\title{
Serotonergic Regulation of Corticoamygdalar Neurons in the Mouse Prelimbic Cortex
}

\author{
Daniel Avesart, Emily K. Stephens ${ }^{\dagger}$ and Allan T. Gulledge* \\ Department of Molecular and Systems Biology, Geisel School of Medicine, Dartmouth College, Hanover, NH, United States
}

Neuromodulatory transmitters, such as serotonin $(5-\mathrm{HT})$, selectively regulate the excitability of subpopulations of cortical projection neurons to gate cortical output to specific target regions. For instance, in the mouse prelimbic cortex, 5-HT selectively excites commissurally projecting (COM) intratelencephalic neurons via activation of 5- $\mathrm{HT}_{2 \mathrm{~A}}$ (2A) receptors, while simultaneously inhibiting, via 5- $\mathrm{HT}_{1 \mathrm{~A}}$ (1A) receptors, corticofugally projecting pyramidal neurons targeting the pons. Here we characterize the physiology, morphology, and serotonergic regulation of corticoamygdalar (CAm) projection neurons in the mouse prelimbic cortex. Layer 5 CAm neurons shared a number of physiological and morphological characteristics with COM neurons, including higher input resistances, smaller HCN-channel mediated responses, and sparser dendritic arbors than corticopontine neurons. Across cortical lamina, CAm neurons also resembled COM neurons in their serotonergic modulation; focally applied 5-HT (100 $\mu \mathrm{M}$;

\section{OPEN ACCESS}

Edited by:

Michael E. Hasselmo,

Boston University, United States

Reviewed by:

Jean-Claude Beique,

University of Ottawa, Canada Akiya Watakabe, RIKEN Center of Brain science (CBS),

Japan

${ }^{*}$ Correspondence: Allan T. Gulledge allan.gulledge@dartmouth.edu

tThese authors have contributed equally to this work.

Received: 17 May 2018

Accepted: 16 July 2018

Published: 07 August 2018

Citation:

Avesar D, Stephens EK and Gulledge AT (2018) Serotonergic Regulation of Corticoamygdalar Neurons in the Mouse Prelimbic Cortex. Front. Neural Circuits 12:63. doi: 10.3389/fncir.2018.00063
1 s) generated 2A-receptor-mediated excitation, or 1A- and 2A-dependent biphasic responses, in ipsilaterally and contralaterally projecting CAm neurons. Serotonergic excitation depended on extrinsic excitatory drive, as 5-HT failed to depolarize CAm neurons from rest, but could enhance the number of action potentials generated by simulated barrages of synaptic input. Finally, using dual tracer injections, we identified double-labeled CAm/COM neurons that displayed primarily excitatory or biphasic responses to $5-\mathrm{HT}$. Overall, our findings reveal that prelimbic CAm neurons in layer 5 overlap, at least partially, with COM neurons, and that neurons projecting to either, or both targets, exhibit 2A-dependent serotonergic excitation. These results suggest that $5-\mathrm{HT}$, acting at $2 \mathrm{~A}$ receptors, may promote cortical output to the amygdala.

Keywords: prefrontal cortex, serotonin, pyramidal neuron, corticoamygdalar projection, 5-HT2A receptor, 5-HT1A receptor

\section{INTRODUCTION}

Output from the neocortex is organized into multiple information channels formed by subclasses of glutamatergic pyramidal neurons defined by their long-distance axonal projections to distinct cortical and subcortical targets. Cortical projection neurons are differentially regulated by a variety of neuromodulatory transmitters through cell-type-specific expression of receptors, signaling proteins, and ionic effectors. For instance, in the mouse prelimbic cortex, acetylcholine (ACh) and serotonin (5-HT) have opposing influences on the excitability of two broad classes of projection neurons: corticofugal neurons, including corticopontine $(\mathrm{CPn})$ neurons, that project to 
deep subcortical targets, and intratelencephalic neurons, including transhemispheric commissural (COM) neurons, that primarily target structures within the telencephalon. Acting at M1-subtype muscarinic receptors, ACh preferentially boosts the excitability of CPn neurons relative to COM neurons (Dembrow et al., 2010; Baker et al., 2018), while 5-HT inhibits CPn neurons via $\mathrm{G}_{\mathrm{i} / \mathrm{o}}$-coupled $5-\mathrm{HT}_{1 \mathrm{~A}}(1 \mathrm{~A})$ receptors, but promotes action potential (AP) generation in COM neurons via $\mathrm{G}_{\mathrm{q}}$-coupled 5- $\mathrm{HT}_{2 \mathrm{~A}}$ (2A) receptors (Avesar and Gulledge, 2012).

Corticoamygdalar (CAm) neurons in the prelimbic cortex that bilaterally target the basolateral amygdala (Mcdonald et al., 1996; Vertes, 2004; Gabbott et al., 2005) represent a less studied, but important, cortical projection that contributes to the expression of learned fear (Stevenson, 2011; Do Monte et al., 2016), and perhaps more generally to coping strategies in the face of external stressors (Varela et al., 2012). For instance, electrical stimulation of the prelimbic cortex promotes expression, and prevents extinction, of conditioned fear (Vidal-Gonzalez et al., 2006), while pharmacological inactivation of the prelimbic cortex impairs expression of conditioned freezing (Corcoran and Quirk, 2007; Sierra-Mercado et al., 2011; Almada et al., 2015). Indeed, unit activity (Burgos-Robles et al., 2009) and field potential oscillations (Dejean et al., 2016; Karalis et al., 2016) in the prelimbic cortex are correlated with animal freezing behavior in conditioned fear paradigms.

Accumulating evidence suggests that fear circuits may be regulated by 5-HT (for reviews, see Bauer, 2015; Bocchio et al., 2016). In rodents, fear conditioning is associated with 5-HT release in the medial prefrontal cortex (mPFC; Yoshioka et al., 1995; Hashimoto et al., 1999), and acute administration of selective serotonin reuptake inhibitors (SSRIs), which act to increase extracellular 5-HT levels, enhances the acquisition and expression of cued fear (Burghardt and Bauer, 2013; Ravinder et al., 2013). Studies targeting specific 5-HT receptor subtypes have found that activation of $1 \mathrm{~A}$ receptors reduces, while $2 \mathrm{~A}$ receptor stimulation enhances, conditioned fear responses. For instance, systemic injection of a 2A agonist (Zhang et al., 2013) enhances freezing in tests of cued and contextual fear memory in mice, while systemic activation of $1 \mathrm{~A}$ receptors reduces freezing behavior in similar tests in rats (Inoue et al., 1996; Ohyama et al., 2016) or mice (Youn et al., 2009). Consistent with these findings, local activation of $1 \mathrm{~A}$ receptors in the prelimbic cortex reduces conditioned fear responses and fear-potentiated startle (Ferreira and Nobre, 2014; Almada et al., 2015), whereas blockade of prelimbic 2A receptors impairs the expression of conditioned fear in a rat strain selectively bred to exhibit heightened anxiety (León et al., 2017). Together, these results suggest that serotonergic activation of $1 \mathrm{~A}$ receptors in the prelimbic cortex may moderate fear responses, while engagement of $2 \mathrm{~A}$ receptors may promote the expression of conditioned fear.

To better understand the physiology of CAm neurons, and the potential role of $5-\mathrm{HT}$ in regulating cortical output to the amygdala, we used retrograde labeling to identify and record from CAm neurons in slices of mouse prelimbic cortex. Here we describe the physiological and morphological properties of these neurons, and their responsivity to 5-HT, in the context of other previously studied prelimbic projection neuron subtypes. Our results demonstrate that prelimbic CAm neurons are in many ways similar to, and overlap with, COM neurons, and suggest that 5 -HT, acting on $2 \mathrm{~A}$ receptors, may promote CAm output to the amygdala.

\section{MATERIALS AND METHODS}

\section{Animals}

Experiments utilized tissue from 8-12-weeks-old male and female C57BL/6J mice (Jackson Laboratories, Bar Harbor, ME, United States) according to methods approved by the Institutional Animal Care and Use Committee of Dartmouth College. Animals had access to food and water ad libitum and were housed on a 12:12 h light:dark cycle.

\section{Retrograde Labeling}

Red or green fluorescent beads (Retrobeads, Lumafluor Inc.) were injected unilaterally into the amygdala to label CAm projection neurons using age appropriate coordinates (Paxinos and Franklin, 2004). In some animals, an additional injection of Retrobeads was made into the ipsilateral mPFC to double-label $\mathrm{CAm} / \mathrm{COM}$ dual-projection neurons. Animals were anesthetized throughout surgeries with vaporized isoflurane $(\sim 2 \%)$. Following craniotomy, a microsyringe was lowered into the brain region of interest, and $300 \mathrm{~nL}$ of undiluted Retrobead solution was injected over a $10 \mathrm{~min}$ period. In some experiments, a nanoject (Drummond scientific) was used to administer the microbeads instead of a microsyringe. Animals were allowed to recover from surgery for at least $72 \mathrm{~h}$ before use in electrophysiological experiments. Locations of dye injections were confirmed post hoc in coronal sections of the amygdala or mPFC.

\section{Slice Preparation}

Following isoflurane anesthesia and decapitation, brains were quickly removed into artificial cerebral spinal fluid (ACSF) containing, in mM: $125 \mathrm{NaCl}, 25 \mathrm{NaHCO}_{3}, 3 \mathrm{KCl}, 1.25 \mathrm{NaH}_{2} \mathrm{PO}_{4}$, $0.5 \mathrm{CaCl}_{2}, 5 \mathrm{MgCl}_{2}$, and 25 glucose, saturated with $95 \% \mathrm{O}_{2} / 5 \%$ $\mathrm{CO}_{2}$. Coronal brain slices (250 $\mu \mathrm{m}$ thick) of the mPFC or amygdala were cut using a Leica VT 1200 slicer and placed in a holding chamber filled with ACSF containing $2 \mathrm{mM} \mathrm{CaCl}_{2}$ and $1 \mathrm{mM} \mathrm{MgCl}_{2}$. Slices were stored at $35^{\circ} \mathrm{C}$ for $\sim 45 \mathrm{~min}$, and then kept at room temperature for up to $8 \mathrm{~h}$ before use in experiments.

\section{Electrophysiology}

Slices were placed in a recording chamber on a fixedstage Olympus BX51WI microscope and continuously perfused with oxygenated ACSF heated to $35-36^{\circ} \mathrm{C}$. Epifluorescence illumination (470 or $530 \mathrm{~nm}$ LEDs) was used to identify CAm or CAm/COM neurons in the mPFC, and wholecell current-clamp recordings were made from bead-labeled neurons using patch pipettes (5-7 $\mathrm{M} \Omega$ ) filled with, in $\mathrm{mM}$ : 135 K-gluconate, $2 \mathrm{NaCl}, 2 \mathrm{MgCl}_{2}, 10 \mathrm{HEPES}, 3 \mathrm{Na}_{2} \mathrm{ATP}$, and $0.3 \mathrm{NaGTP}$ (pH 7.2 with $\mathrm{KOH}$ ). Data were acquired with Axograph software (Axograph Company) using BVC700 amplifiers (Dagan Corporation) and ITC-18 digitizers 
(HEKA Instruments). Membrane potentials were sampled at $25 \mathrm{kHz}$, filtered at $10 \mathrm{kHz}$, and corrected for a liquid junction potential of $+12 \mathrm{mV}$. Hyperpolarization-activated cyclic-nucleotide gated ( $\mathrm{HCN}$ )-channel-mediated rebound (i.e., "sag" potentials) were measured as the percent difference in the peak hyperpolarization and the steady state voltage (both relative to resting membrane potentials [RMPs]) in response to negative current injection set to hyperpolarize the neuron by approximately $20 \mathrm{mV}$.

5-HT $(100 \mu \mathrm{M})$ was dissolved in ACSF and loaded into patch pipettes placed $\sim 50 \mu \mathrm{m}$ from targeted somata. Neurons were classified as 5-HT-inhibited, -excited, -biphasic, or -nonresponsive based on their response to focal application of 5HT, which was delivered for 1 s at $\sim 15$ PSI during periods of AP generation $(\sim 5 \mathrm{~Hz})$ evoked by somatic DC current injection. Serotonergic inhibition was quantified as the duration of cessation of AP generation, while excitatory responses were quantified as the percent increase in the mean instantaneous spike frequency (ISF; the inverse of the time interval between each AP) measured over the $500 \mathrm{~ms}$ following the peak post5-HT ISF, relative to the mean pre-5-HT ISF baseline (10 s). Biphasic 5-HT responses were defined as responses having both brief inhibition (lasting at least 10 times the average baseline interspike interval) and a subsequent increase in ISF of at least $1 \mathrm{~Hz}$ above baseline levels. Mean "population" responses to 5-HT during DC-current-induced AP firing were made by resampling individual ISF plots at $2 \mathrm{~Hz}$ and then averaging those ISF plots across tested neurons. In some experiments, 5-HT receptors were selectively blocked with 1A (WAY 100635, 30 nM; SigmaAldrich) and/or 2A (MDL 11939, 500 nM; Tocris) receptor antagonists.
In some experiments, somatic current injections were used to simulate excitatory synaptic input, as previously described (Stephens et al., 2014). Because of intrinsic cell-to-cell variability in input resistance and excitability, for each neuron, the synaptic waveform was scaled in amplitude to evoke $\sim 8$ APs during baseline trials. The simulated synaptic current was then delivered 29 times at $3 \mathrm{~s}$ intervals, the exception being the sixth trial which was delayed $3 \mathrm{~s}$ due to application of 5-HT $(100 \mu \mathrm{M}, 1 \mathrm{~s})$.

\section{Morphological Analyses and Single-Cell Imaging}

In some experiments, biocytin (7 mg/ml; Tocris) was included in the patch-pipette solution. Following recordings, slices were placed in a phosphate buffered saline (PBS) solution containing $4 \%$ paraformaldehyde for $24 \mathrm{~h}$. Slices were then rinsed three times for $15 \mathrm{~min}$ in PBS and placed in a PBS solution containing $0.25 \%$ Triton X-100 and avidin conjugated to either Alexa Fluor ${ }^{\circledR}$ 594 or Alexa Fluor ${ }^{\circledR}-488(20 \mu \mathrm{g} / \mathrm{ml}$; Invitrogen $)$ for $24 \mathrm{~h}$. Slices were then rinsed with PBS three times for fifteen minutes each, dried, and mounted on slides in FluorSave (EMD Chemicals). Cells were imaged using a two-photon microscope (Bruker Corporation) or a Zeiss LSM 510 confocal microscope (Carl Zeiss). Data from COM and CPn neurons used for comparisons were previously published in Avesar and Gulledge (2012) and Stephens et al. (2014). Morphological measurements included somatic distance from the pia, maximum horizontal width of the apical tuft, the number of dendritic branch points in the tuft, and the number of primary and oblique dendrites. Tracings of neurons were made using NeuronJ from z-stack projections.

TABLE 1| Physiological properties of layer 5 neurons.

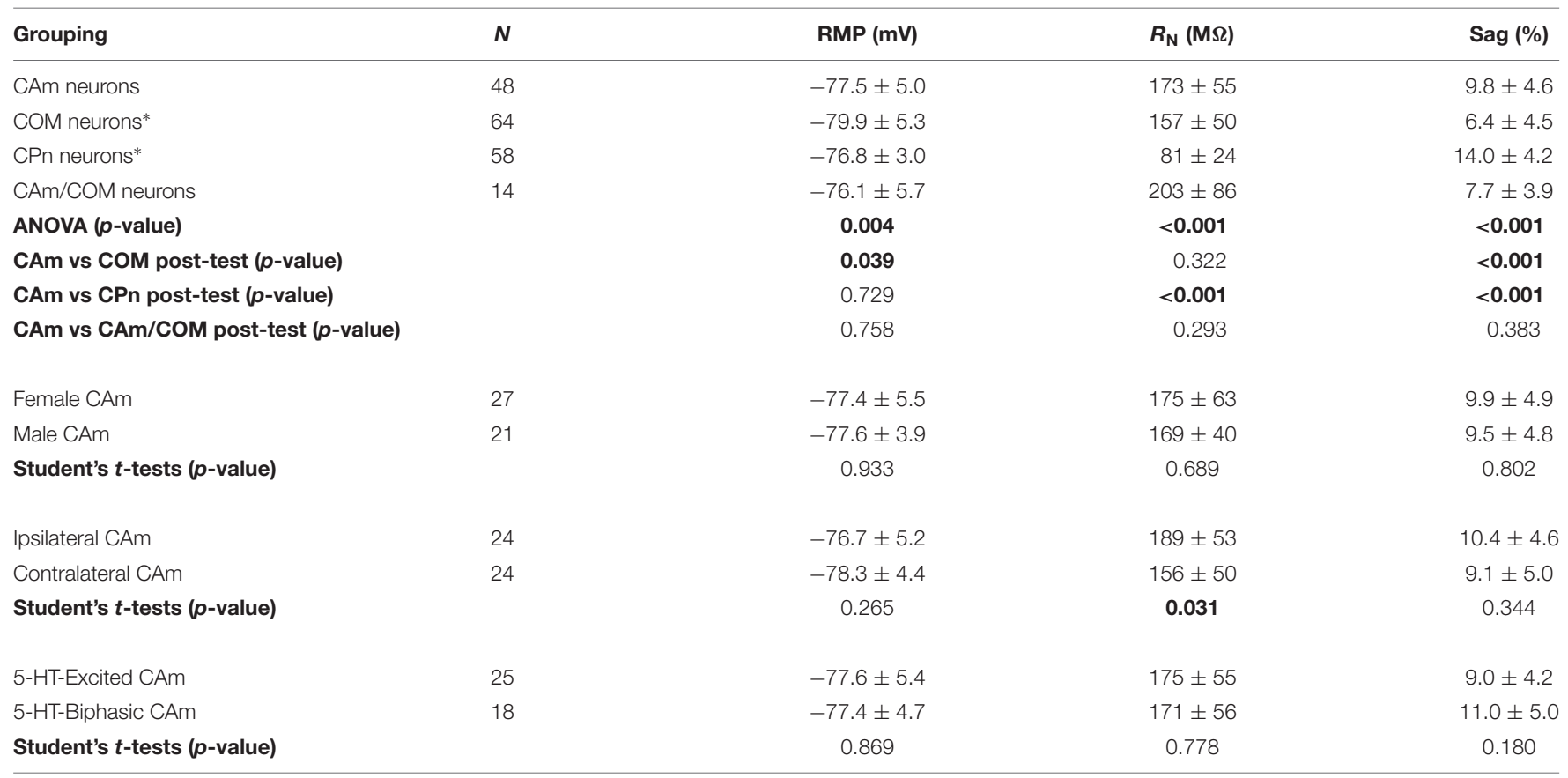

Data presented as means \pm standard deviations. *Data from Avesar and Gulledge (2012) and Stephens et al. (2014). P-values < 0.05 are in boldface type. 

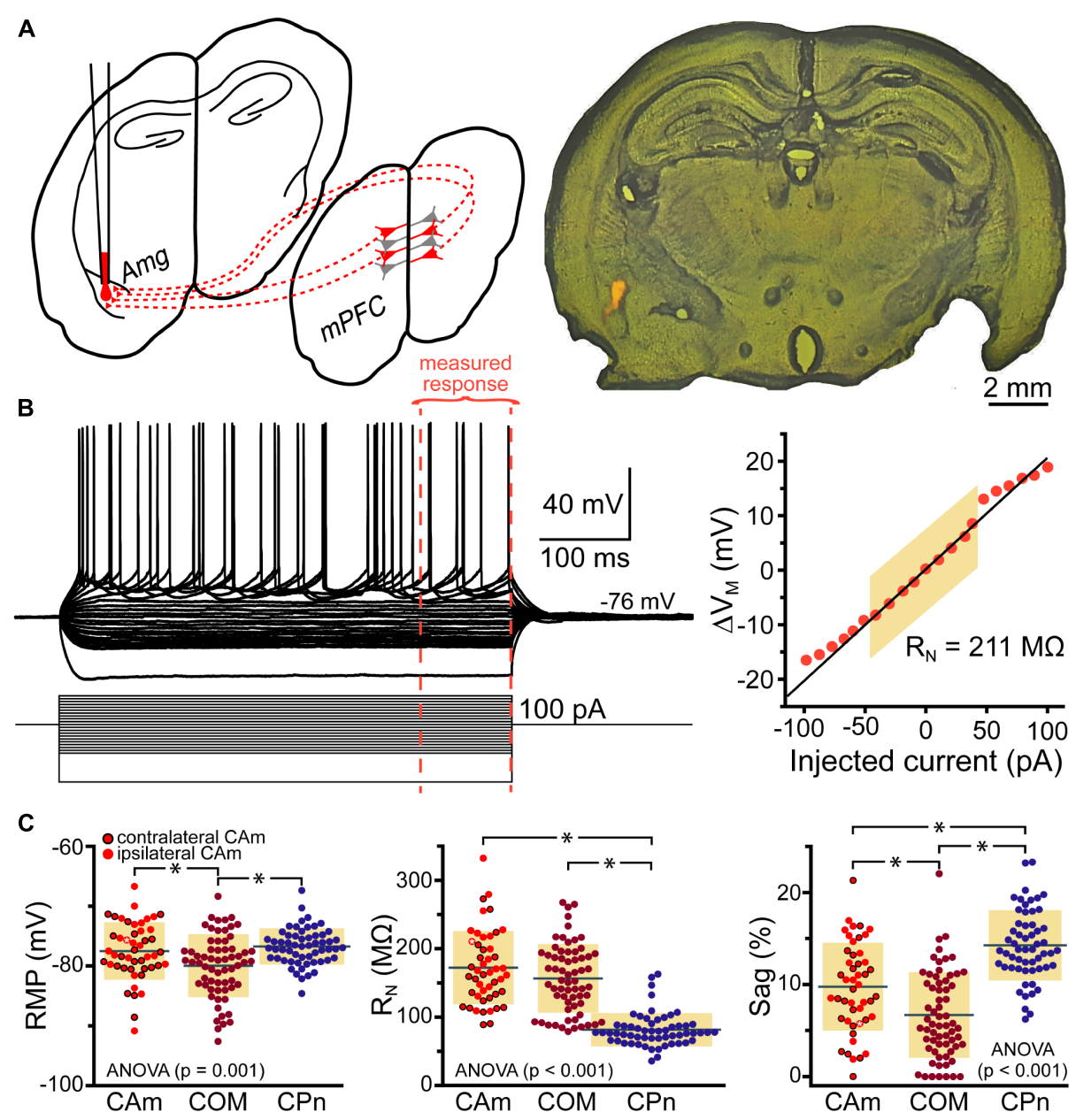

FIGURE 1 | Physiological properties of corticoamygdalar (CAm) neurons. (A) Left - Schematic of bilateral retrograde labeling of CAm neurons in the medial prefrontal cortex. Right - a coronal section of a mouse brain showing the amygdala containing fluorescent retrograde tracers. (B) Voltage traces (top left) of an L5 CAm neuron in response to a family of positive and negative current injections (bottom left). Red dashed lines show the final 100 ms of the voltage responses. At right is a plot of the voltage-current relationship as measured across the final $100 \mathrm{~ms}$ of the voltage responses. The shaded area shows the linear region of the relationship that was used to calculate input resistance $\left(\mathrm{R}_{\mathrm{N}}\right)$. (C) Plots of individual measurements of resting membrane potential (RMP; left), $\mathrm{R}_{\mathrm{N}}$ (middle), and HCN-channel dependent voltage sag (right) for CAm $(n=48)$, COM $(n=64)$, and CPn $(n=58)$ neurons. Black horizontal lines indicate means and shaded regions indicate \pm 1 standard deviation. Symbols with black borders indicate contralateral CAm neurons. Symbols with white asterisks indicate the data from the cell shown in B. Data for COM and CPn neurons aggregated from Avesar and Gulledge (2012) and Stephens et al. (2014). Black asterisks indicate $p<0.05$, Student's $t$-test.

\section{Statistical Analyses}

Unless otherwise noted, all data are presented as means \pm standard deviations. Comparisons across cell groups utilized one-way ANOVAs with Šidák post-tests, while comparisons within groups was accomplished using two-tailed Student's $t$-tests (paired or unpaired), or repeated measures

TABLE 2 | Comparison of morphological properties of layer 5 projection neurons.

\begin{tabular}{|c|c|c|c|c|c|}
\hline Grouping & $n$ & Depth from pia $(\mu \mathrm{m})$ & \# Primary dendrites & \# Oblique dendrites & Apical tuft width $(\mu \mathrm{m})$ \\
\hline CAm neurons & 21 & $335 \pm 78^{\dagger}$ & $7.1 \pm 1.5$ & $7.4 \pm 4.1$ & $289 \pm 126$ \\
\hline COM neurons* & 10 & $332 \pm 58$ & $6.7 \pm 2.4$ & $4.6 \pm 2.3$ & $211 \pm 95$ \\
\hline CPn neurons* & 10 & $405 \pm 41$ & $9.2 \pm 2.8$ & $13.3 \pm 3.5$ & $302 \pm 96$ \\
\hline \multicolumn{2}{|c|}{ ANOVA ( $p$-value) } & 0.026 & 0.018 & $<0.001$ & 0.140 \\
\hline \multicolumn{2}{|c|}{ CAm vs COM post-test (p-value) } & 0.999 & 0.922 & 0.165 & \\
\hline \multicolumn{2}{|c|}{ CAm vs CPn post-test (p-value) } & 0.043 & 0.030 & 0.002 & \\
\hline
\end{tabular}

Data presented as means \pm standard deviations. *Data from Avesar and Gulledge (2012). ${ }^{\dagger} n=19$ for depth from pia. $P$-values < 0.05 are in boldface type. 
ANOVA, as appropriate. Comparisons of response proportions between groups utilized a Fisher's exact test. Significance was defined as $p<0.05$.

\section{RESULTS}

\section{Physiological and Morphological Characteristics of CAm Neurons}

Retrograde-labeled pyramidal neurons projecting to either the ipsilateral (iCAm neurons; $n=24$ ) or contralateral (cCAm neurons; $n=24$ ) amygdala were targeted for whole-cell recordings in layer 5 of the mouse prelimbic cortex. For each neuron, measurements were made of RMP, input resistance $\left(\mathrm{R}_{\mathrm{N}}\right)$, and HCN-channel-mediated rebound "sag" potentials (\% sag; see section "Materials and Methods"; Table 1 and Figure 1). CAm neuron physiology was not sex-dependent (Table 1), and RMP and $\mathrm{HCN}$ responses were not dependent on the laterality of amygdalar projection (Table 1). However, ipsilaterally projecting CAm neurons had a significantly higher input resistance than contralateral CAm neurons ( $p=0.031$; Student's $t$-test; Table 1). The physiological properties of CAm neurons, as a whole, were somewhat distinct from those previously observed in layer 5 COM and CPn neurons (Avesar and Gulledge, 2012; Stephens et al., 2014). While CAm neurons resembled COM neurons in having larger $\mathrm{R}_{\mathrm{N}} \mathrm{s}$ (at $172 \pm 54 \mathrm{M} \Omega ; n=48 ; p<0.001$; Student's $t$-test) and smaller HCN-dependent sag responses (at $9.8 \pm 4.8 \%$; $p<0.001)$ than CPn neurons $(n=58$; Table 1$)$, the RMPs of CAm neurons $(-78 \pm 5 \mathrm{mV})$ were similar to those in CPn neurons $(-77 \pm 3 \mathrm{mV} ; p=0.73)$, and more depolarized than those in COM neurons $(-80 \pm 5 \mathrm{mV} ; n=64 ; p=0.039)$, likely reflecting their enhanced sag potentials relative to those of COM neurons $(6.4 \pm 4.6 \% ; p<0.001$; see Table 1 and Figure 1C).

Morphologically, layer 5 CAm neurons $(n=21)$ resembled $\mathrm{COM}$, rather than $\mathrm{CPn}$, neurons (Figure 2). Both CAm and COM neurons had fewer primary (means of $7.1 \pm 1.5$ and $6.7 \pm 2.4$, respectively; $p=0.02$; ANOVA) and oblique (means of $7.4 \pm 4.1$ and $4.6 \pm 2.3$, respectively; $p<0.001$; ANOVA) dendrites than did CPn neurons ( $n=10$; Table 2 and Figure 2B). While cells of all projection subtypes exhibited similar apical tuft widths (e.g., $289 \pm 126 \mu \mathrm{m}$ for CAm neurons; $p=0.14$, ANOVA), CAm and COM $(n=10)$ neuron somata tended to be slightly more superficial (at $339 \pm 18$ and $332 \pm 18 \mu \mathrm{m}$ from the pia; $p=0.029$ and 0.010 ; Sidák post-tests for CAm and COM neurons, respectively, vs CPn neurons) than the somata of CPn neurons (405 $\pm 13 \mu \mathrm{m}$; Figure 2B and Table 2). Thus, while CAm neurons were in some ways distinct from COM and CPn neurons (e.g., in their HCN-mediated responses), their overall physiological and morphological characteristics were more similar to COM neurons.

\section{CAm Neurons Are Excited by 5-HT via 2A Receptors}

Corticoamygdalar neurons also resembled COM neurons in their responses to focal application of 5-HT during periods

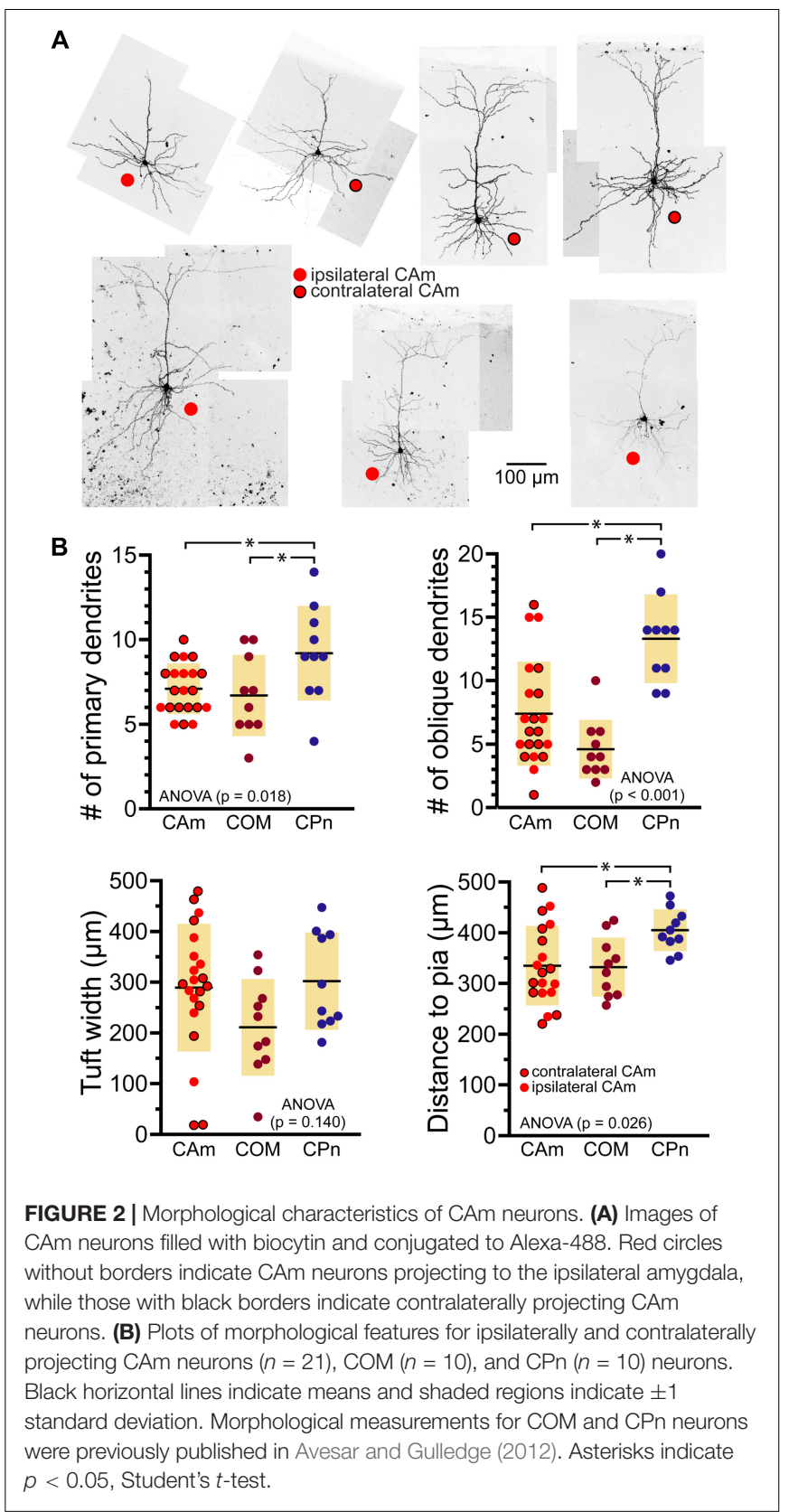

of current-induced AP generation (Figure 3 and Table 3). 5 -HT enhanced the frequency of AP generation in 25 of 48 CAm neurons (52\%), and generated biphasic responses in 18 CAm neurons (38\%; Figures 3A,B). Four CAm neurons were non-responsive to 5-HT, and one CAm neuron exhibited a purely inhibitory response to 5-HT. The proportions of CAm excited and biphasic neurons were statistically similar in 24 iCAm and 24 cCAm neurons $(p=0.36$; Fisher's exact test; Figure 3C), suggesting that 5-HT influences CAm neurons independently of the laterality of their amygdalar projections. Indeed, the magnitudes of serotonergic excitation were similar between iCAm $(n=11)$ and cCAm $(n=14)$ 5-HT-excited neurons (mean excitations of $84 \pm 63$ and $121 \pm 115 \%$, 
A

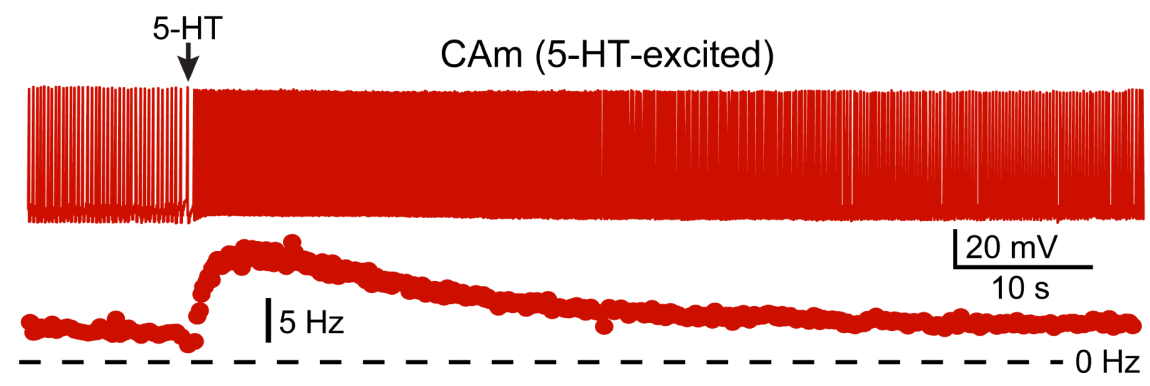

B
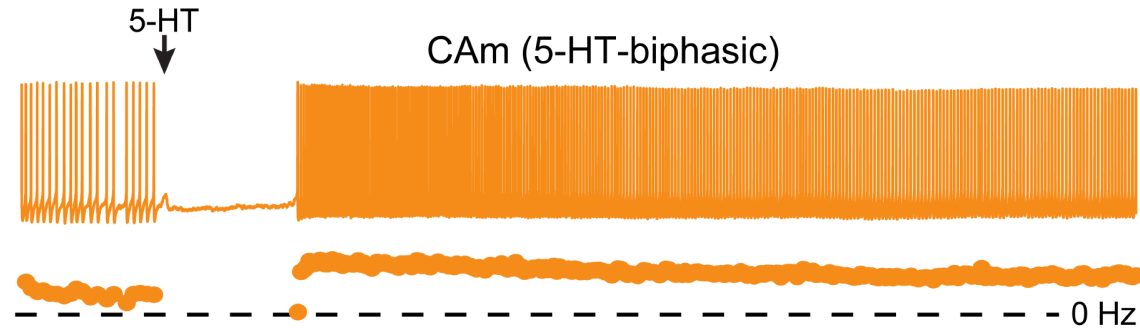

C

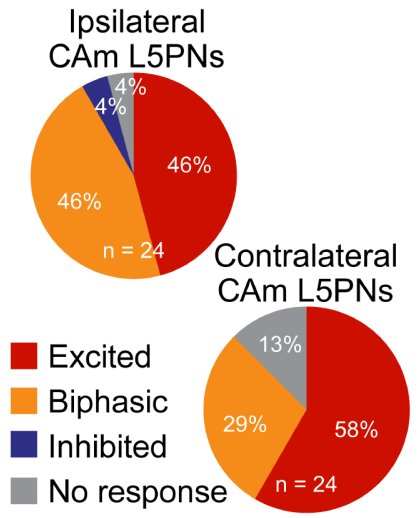

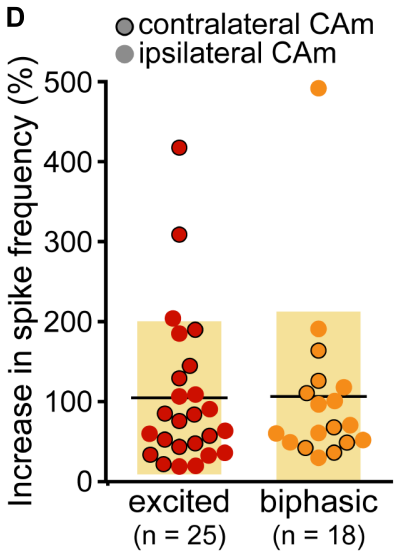

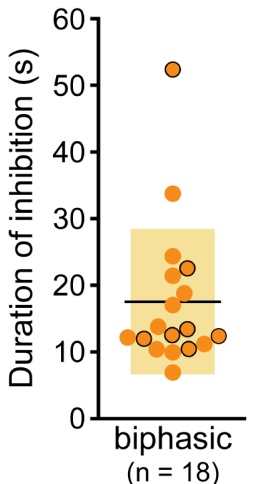

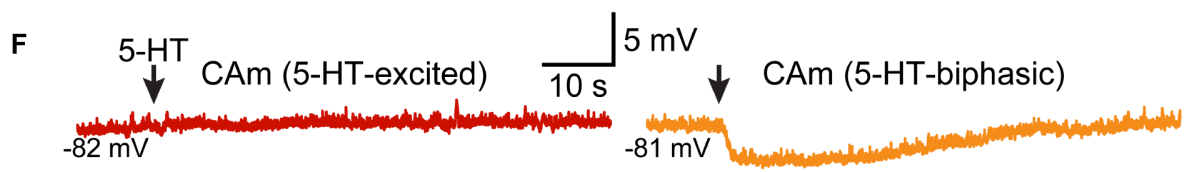

FIGURE 3 | 5-HT excites CAm neurons. (A) Voltage trace (top) and plot of instantaneous spike frequency (ISF; bottom) of action potentials in a CAm neuron during prolonged somatic DC current injection. Focal application of $100 \mu \mathrm{M} 5-\mathrm{HT}$ (1 s; arrow) enhanced action potential frequency in this neuron. Dashed line indicates $0 \mathrm{~Hz}$. (B) Voltage trace (top) and plot of ISF (bottom) over time in a CAm neuron exhibiting a biphasic (inhibition followed by excitation) response to focal application of 5-HT (arrow). (C) Graphs showing the proportions of ipsilaterally and contralaterally projecting layer 5 CAm neurons that exhibited excitatory (red), biphasic (yellow), or inhibitory (blue) responses to $5-\mathrm{HT}$, or no response at all (gray). (D) Plots of the magnitudes of excitatory responses to 5-HT (\% relative to baseline firing rates) for ipsilaterally (non-bordered symbols) and contralaterally (black-bordered symbols) projecting 5-HT-excited (red) and 5-HT-biphasic (yellow) CAm neurons. (E) Plots of the duration of 5-HT-induced spike cessation in ipsilaterally and contralaterally projecting 5-HT-biphasic CAm neurons. (F) Voltage traces of a 5-HT-excited CAm neuron (red, left) and a 5-HT-biphasic neuron (yellow, right) during 5-HT application at resting membrane potentials (-82 and -81 mV, respectively).

respectively; $p=0.32$; Student's $t$-test). Excitatory responses in iCAm $(n=11)$ and cCAm $(n=7)$ 5-HT-biphasic neurons (mean excitations of $120 \pm 131$ and $85 \pm 49 \%$, respectively; Figure 3D) were also similar to each other $(p=0.44)$ and to the magnitude of excitation observed across all 5HT-excited CAm neurons (98 $\pm 71 \% ; n=25 ; p=0.77$, ANOVA). Further, serotonergic biphasic inhibitions in iCAm and CCAm neurons were of similar durations (at $16 \pm 8$ and $19 \pm 5 \mathrm{~ms}$, respectively; $p=0.64$; Student's $t$-test;
Figure 3E). These results suggest that 5-HT responses in CAm neurons are not dependent on projection laterality. Additionally, 5 -HT responses in CAm neurons were not sex-dependent (Table 3).

5 -HT responses in layer $5 \mathrm{CAm}$ neurons were qualitatively and quantitatively similar (in both proportions and magnitudes) to 5-HT responses previously reported in COM neurons (see Table 3). Further, as previously reported in COM neurons (Stephens et al., 2014), serotonergic excitation of CAm neurons 
required additional depolarizing drive, as 5-HT failed to depolarize 5 -HT-excited CAm $(n=8)$ or 5 -HT-biphasic $(n=7)$ neurons from RMPs (Figure 3F). In 5-HT-excited CAm neurons, the mean change in membrane potential relative to RMPs was $+0.1 \pm 0.5 \mathrm{mV}$ ( $p=0.54$, paired Student's $t$-test). In 5-HTbiphasic CAm neurons, focal application of 5-HT at RMPs led to transient hyperpolarizations (of $3.5 \pm 0.9 \mathrm{mV} ; p<0.001$ ) that lasted $27 \pm 11 \mathrm{~s}$, but no subsequent depolarizations (mean peak membrane potential relative to RMPs was $+0.4 \pm 1.1 \mathrm{mV}$; $p=0.43$ ). Thus, serotonergic stimulation, on its own, was not sufficient to excite CAm neurons.

Serotonergic excitation of COM neurons is mediated by $2 \mathrm{~A}$ receptors, whereas biphasic responses involve both $1 \mathrm{~A}$-mediated inhibition and 2A-driven excitation (Avesar and Gulledge, 2012; Stephens et al., 2014). To test whether $2 \mathrm{~A}$ receptors also mediate serotonergic excitation of CAm neurons, we measured 5-HT responses in 5-HT-excited and 5-HT-biphasic CAm neurons before and after bath application of the selective $2 \mathrm{~A}$ receptor antagonist MDL 11939 (500 nM). In both 5-HT-excited $(n=8)$ and 5-HT-biphasic $(n=7)$ CAm neurons, MDL blocked serotonergic excitation (Figure 4). In a subset of 5-HT-biphasic neurons $(n=3)$, the inhibitory portion of biphasic responses was blocked by WAY 100635 (30 nM), a 1A antagonist (Figure 4C). These results confirm that serotonergic responses in prelimbic CAm neurons are mediated by the same receptor subtypes that gate serotonergic excitation and inhibition in COM and
CPn neurons (Avesar and Gulledge, 2012; Stephens et al., 2014).

\section{5-HT Enhances CAm Neuron Output to Simulated Synaptic Input}

To test whether 5-HT can functionally enhance the output of CAm neurons driven by a more physiological stimulus, we repeatedly delivered a barrage of simulated synaptic current (see section "Materials and Methods") over 29 trials (3 s inter-trial intervals), and measured the number of APs generated in each trial, as well as the RMP just prior to the barrage (Figure 5). Following the initial five baseline trials, 5-HT was applied for $1 \mathrm{~s}$ before synaptic barrages were resumed for 24 additional trials. In 5-HT-excited CAm neurons $(n=14)$, simulated synaptic barrages generated a mean of $7.6 \pm 1.2$ APs during the five baseline trials. Following 5-HT application, there was an increase of $2.4 \pm 1.9$ APs per trial, bringing the mean response to $10.0 \pm 2.1$ APs across the subsequent five trials $(p<0.001$; repeated measures ANOVA; Figure 5B, red symbols). Conversely, 5-HT had little impact on AP output in biphasic CAm neurons (mean change of $-1.6 \pm 4$ APs, relative to their baseline response of $8.9 \pm 1.7$ APs; $p=0.31$, Figure 5B, yellow symbols). Since 5-HT generated a small, but significant $(p=0.024)$, depolarization of the RMP in 5-HT-excited CAm neurons (peaking with a mean of $1.2 \pm 1.3 \mathrm{mV}$ on trial 11 , about $6 \mathrm{~s}$ after 5 -HT application,

TABLE 3 | Serotonergic responses in CAm neurons.

\begin{tabular}{|c|c|c|c|c|}
\hline Grouping & $n$ & 5-HT response & Excitation ( $\%$ increase in ISF) & Duration of inhibition (s) \\
\hline \multirow[t]{2}{*}{ L5 COM neurons* } & 43 & Excited & $142 \pm 101$ & NA \\
\hline & 21 & Biphasic & $153 \pm 78$ & $17 \pm 9$ \\
\hline \multirow[t]{2}{*}{ L5 CAm neurons } & 25 & Excited & $98 \pm 97$ & NA \\
\hline & 18 & Biphasic & $111 \pm 102$ & $18 \pm 11$ \\
\hline \multirow[t]{2}{*}{ L2/3 CAm neurons } & 7 & Excited & $93 \pm 71$ & NA \\
\hline & 6 & Biphasic & $148 \pm 101$ & $27 \pm 19$ \\
\hline \multirow[t]{2}{*}{ L5 CAm/COM neurons } & 9 & Excited & $54 \pm 34$ & NA \\
\hline & 4 & Biphasic & $70 \pm 45$ & $16 \pm 7$ \\
\hline ANOVA & & & 0.077 & 0.249 \\
\hline Ipsilateral L5 CAm & 11 & Excited & $84 \pm 63$ & NA \\
\hline Contralateral L5 CAm & 14 & Excited & $121 \pm 115$ & NA \\
\hline Student's $t$-test ( $p$-value) & & & 0.321 & \\
\hline Ipsilateral L5 CAm & 11 & Biphasic & $120 \pm 131$ & $16 \pm 8$ \\
\hline Contralateral L5 CAm & 7 & Biphasic & $85 \pm 49$ & $19 \pm 15$ \\
\hline Student's $t$-tests ( $p$-value) & & & 0.436 & 0.643 \\
\hline Female L5 CAm & 15 & Excited & $108 \pm 83$ & NA \\
\hline Male L5 CAm & 10 & Excited & $99 \pm 116$ & NA \\
\hline Student's $t$-test ( $p$-value) & & & 0.837 & \\
\hline Female L5 CAm & 9 & Biphasic & $120 \pm 142$ & $20 \pm 15$ \\
\hline Male L5 CAm & 9 & Biphasic & $93 \pm 58$ & $15 \pm 5$ \\
\hline Student's $t$-tests ( $p$-value) & & & 0.611 & 0.315 \\
\hline
\end{tabular}

Data presented as means \pm standard deviations. *Data from Avesar and Gulledge (2012) and Stephens et al. (2014). NA, not applicable. 

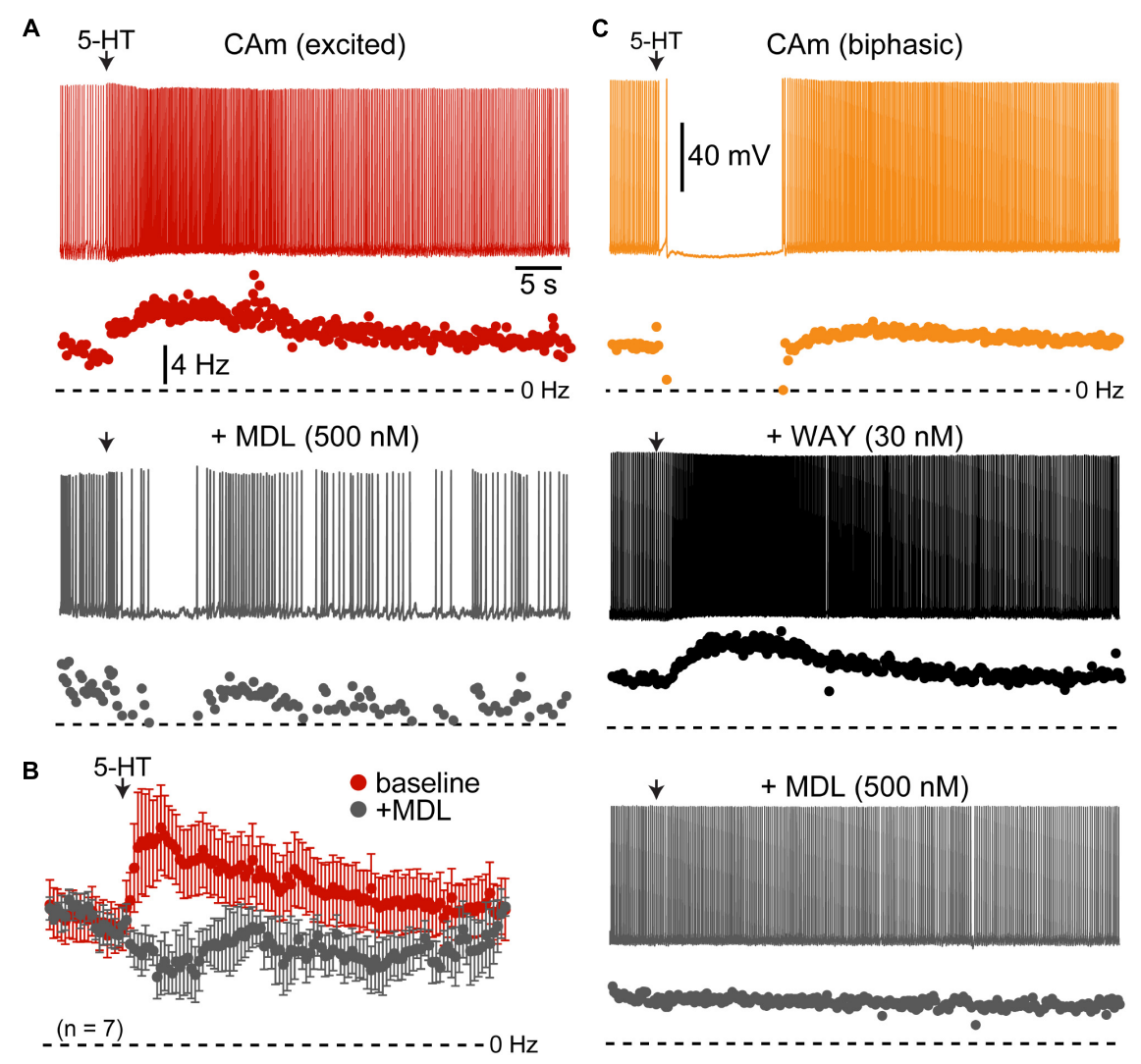

FIGURE 4 | Serotonergic responses in CAm neurons are mediated by $1 \mathrm{~A}$ and $2 \mathrm{~A}$ receptors. (A) Excitatory serotonergic response of a layer 5 CAm neuron (red voltage trace and plot of instantaneous spike frequency [ISF], top) that was subsequently blocked by bath application of the 2A antagonist MDL 11939 (500 nM; gray voltage trace and ISF plot). Arrows indicate timing of 5-HT application. (B) Population response (mean ISF \pm SEM) to 5-HT for seven 5-HT-excitd CAm neurons before (red) and after (gray) addition of $500 \mathrm{nM}$ of MDL 11939. (C) Voltage traces (top) and ISF plots (bottom) showing responses to 5-HT (arrow) in a biphasic layer $5 \mathrm{CAm}$ neuron under baseline conditions (yellow trace/plot) and after sequential addition of the 1A receptor antagonist WAY 100635 (30 nM; black trace/plot) and the 2A antagonist MDL 11939 (500 nM; gray trace/plot).

and only after application of suprathreshold current input), in a subset of 5-HT-excited neurons $(n=8)$ we tested whether depolarization alone could account for the additional APs produced after 5-HT application. In these control experiments, in which $7.1 \pm 1.5$ spikes were generated in baseline trials, DC current was used to continuously depolarize neurons by $1.8 \pm 0.7 \mathrm{mV}(p<0.001)$ during the remaining 24 trials. This depolarization alone failed to significantly increase the number of APs generated by the simulated synaptic input (mean of $7.7 \pm 2.2$ APs; $p=0.12$ ). Together, these results confirm previous findings in COM neurons (Stephens et al., 2014), and suggest that 5HT will preferentially enhance the output of 5-HT-excited CAm neurons that are receiving coincident suprathreshold synaptic drive.

\section{5-HT Generates Biphasic and Excitatory Responses in L2/3 CAm Neurons}

Although our study focuses on layer 5 pyramidal neurons, CAm neurons are present across cortical lamina (Little and Carter, 2013; Song et al., 2015; Cheriyan et al., 2016). To determine whether 5-HT regulates the excitability of superficial CAm neurons, we recorded from Retrobead-labeled CAm neurons in layer $2 / 3$ of the prelimbic cortex $(n=13)$. Layer $2 / 3$ CAm neurons had physiological properties comparable to those in layer 5 , with mean RMPs of $-77 \pm 7 \mathrm{mV}$ ( $p=0.98$ vs 48 layer 5 CAm; Student's $t$-test), input resistances of $208 \pm 82 \mathrm{M} \Omega(p=0.61$ vs layer 5 CAm), and sag potentials of $7.3 \pm 3.8 \%(p=0.30$ vs layer 5 CAm). Layer 2/3 CAm neurons exhibited excitatory $(n=7)$ or biphasic $(n=6)$ responses to focally applied 5-HT (Figure 6 and Table 3 ) that occurred in proportions ( $p=1.00$; Fisher's exact test) and magnitudes (mean increases above baseline ISF of $93 \pm 71$ and $148 \pm 101 \%$ for 5-HT-excited and 5-HT-biphasic L2/3 CAm neurons, respectively; $p=0.22$ and 0.91 , when compared to layer 5 CAm neurons) consistent with those observed in layer 5 CAm and COM neurons. Thus, serotonergic regulation of CAm neuron excitability appears to be conserved across cortical layers in the prelimbic cortex.

\section{CAm Neurons Overlap With COM Neurons}

Based on their morphological and physiological similarities, we hypothesized that CAm and COM neurons may represent 


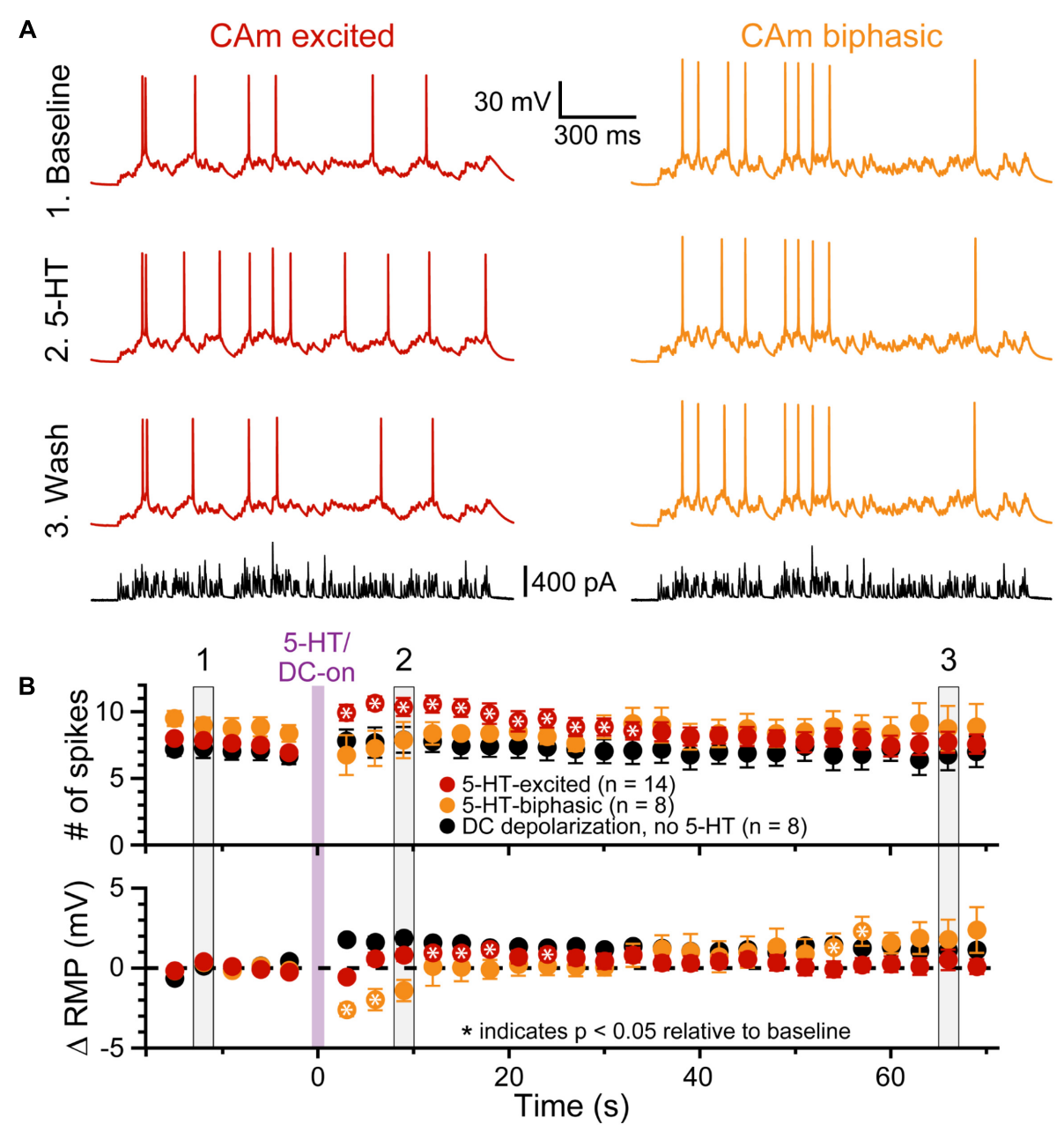

FIGURE 5 | 5-HT enhances CAm neuron output in response to simulated synaptic input. (A) Voltage traces from 5-HT-excited (red traces) and 5-HT-biphasic (yellow traces) CAm neurons in response to a simulated barrage of synaptic input (black traces) delivered in (1) baseline conditions (top traces), (2) after focal application of 5-HT (middle traces), and (3) about 1 min later (lower voltage traces). (B) Population responses (means \pm SEMs) for 5 -HT-excited (red symbols; $n=14$ ) and 5-HT-biphasic CAm neurons (yellow symbols; $n=8$ ). Black symbols show data from a subset of 5-HT-excited neurons $(n=8)$ receiving DC current, instead of 5 -HT, to depolarize the cells $1-2 \mathrm{mV}$ following five baseline applications of simulated synaptic current. The mean number of action potentials in response to the simulated synaptic barrage is plotted over time in the top graph, while the lower graph plots the mean changes in resting membrane potentials (RMPs), relative to baseline values, as measured for each trial immediately before simulated synaptic current application. Asterisks indicates $p<0.05$ relative to baseline measurements from repeated measures ANOVA. Gray vertical bars (1, 2, and 3) indicate the trials represented in $\mathbf{A}$.

overlapping populations of layer 5 neurons in the mPFC. We tested this hypothesis by injecting Retrobeads unilaterally into the contralateral amygdala and the $\mathrm{mPFC}$ to label $\mathrm{CAm} / \mathrm{COM}$ dual projection neurons (Figure 7A). While $\mathrm{CAm} / \mathrm{COM}$ neurons were much less numerous than single-labeled COM and CAm neurons, we recorded from 14 dual-labeled neurons, finding that the majority $(64 \%, n=9 / 14)$ were excited by $5-\mathrm{HT}$, with an additional 29\% $(n=4 / 14)$ exhibiting biphasic responses to 5-HT. One CAm/COM double-labeled neuron (7\%) exhibited a purely inhibitory response to 5-HT (Figures 7B,C). The magnitudes of excitatory responses in CAm/COM 5-HT-excited (54 $\pm 34 \%)$ and 5-HT-biphasic $(70 \pm 45 \%)$ neurons (Figure 7D and Table 3) were statistically similar to those observed in single-labeled 5HT-excited CAm neurons $(p=0.13$ and 0.51 , for CAm/COM 5-HT-excited and 5-HT-biphasic neurons, respectively; Student's $t$-tests). Similarly, the durations of inhibitory responses in CAm/COM 5-HT-biphasic neurons $(19 \pm 5$ s) were similar to those in single-labeled CAm neurons $(p=0.81)$. The physiological properties of $\mathrm{CAm} / \mathrm{COM}$ neurons were also similar to those observed in single-labeled CAm neurons (Table 1). These data demonstrate that, in the mouse prelimbic cortex, layer $5 \mathrm{CAm}$ and $\mathrm{COM}$ neurons are overlapping neuron populations that share $2 \mathrm{~A}$-mediated serotonergic excitation. However, because retrograde labeling with beads does not label all projection neurons to a given target region, and has variable efficiency animal-to-animal, it was not possible to calculate the extent of $\mathrm{CAm} / \mathrm{COM}$ overlap. Therefore, it remains possible that layer $5 \mathrm{CAm}$ and COM cells largely represent a single, but diverse, population of intratelencephalic neurons. 


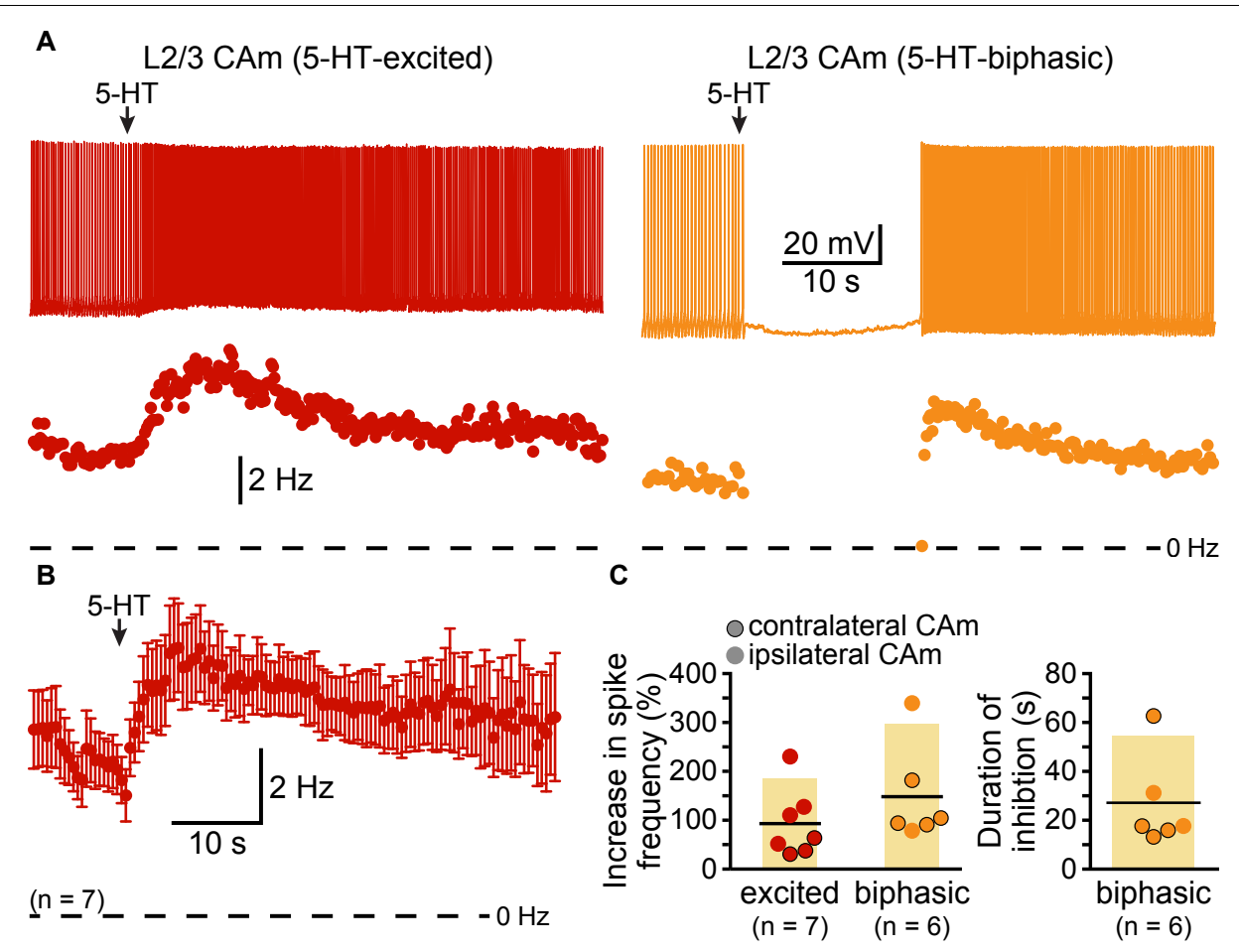

FIGURE 6 | Serotonergic regulation of layer 2/3 CAm neurons. (A) Voltage traces (top) and plots of instantaneous spike frequencies (ISFs; bottom) of action potentials in two CAm neurons in layer $2 / 3$ of the prelimbic cortex experiencing continuous somatic DC current injection. Focal application of 100 MM 5 -HT (1 s; arrows) increased the frequency of action potential genesis in one neuron (left), and generated a biphasic response in the other neuron (right). (B) Population response (mean ISF \pm SEM) to 5-HT for seven 5-HT-excited layer 2/3 CAm neurons. (C) Plots of the increase in spike frequency in response to 5-HT (\% relative to baseline firing rates) for ipsilaterally (non-bordered symbols) and contralaterally (black-bordered symbols) projecting 5-HT-excited (red) and 5-HT-biphasic (yellow) layer 2/3 CAm neurons (left), and of the duration of 5-HT-induced spike-cessation in 5-HT-biphasic layer 2/3 CAm neurons (right).

\section{DISCUSSION}

Cortical projections to the amygdala convey top-down control of emotional responses, including fear behaviors (for review, see Courtin et al., 2013). Activity in the prelimbic cortex, likely conveyed to the amygdala via CAm neurons (Stevenson, 2011; Karalis et al., 2016), facilitates emotional responses to conditioned cues, most prominently freezing behavior (Corcoran and Quirk, 2007; Burgos-Robles et al., 2009; Dejean et al., 2016), but also active avoidance (Bravo-Rivera et al., 2014, 2015), suggesting a more generalized role of the prelimbic cortex in goal-directed behaviors and behavioral flexibility (for reviews, see Ragozzino, 2007; Euston et al., 2012; Orsini et al., 2015). Our findings demonstrate that layer 5 CAm neurons in the mouse prelimbic cortex share morphological and physiological properties with COM neurons. Like COM neurons, CAm neurons have sparser dendritic trees, higher input resistances, and less HCN-channel-mediated "sag" potentials than typically observed in neighboring corticofugal (e.g., CPn) neurons. Although the magnitude of $\mathrm{HCN}$-mediated sag potentials in CAm neurons were somewhat larger (and RMPs more depolarized) than reported in COM neurons (Table 1 and Figure 1C), we identified double-labeled CAm/COM neurons following dual injections of red and green beads in the amygdala and contralateral cortex, suggesting that layer 5 CAm and COM neuron populations overlap, at least partially, although the extent of such overlap was not determinable in our study. While we did not test for overlap among layer 2/3 CAm and COM neurons, our findings in layer 5 contrasts with the discrete, non-overlapping, ipsilateral CAm and COM populations reported in layer 2 of the mPFC in Swiss Webster mice (Little and Carter, 2013). This disparity may reflect differences in the laterality of CAm injections (ipsilateral vs contralateral), differences across animal strain, or functional diversity in CAm projections across cortical lamina. However, regardless of population overlap, our study, and that by Little and Carter (2013), agrees in finding that CAm and COM neurons are broadly similar in their physiological and morphological properties.

A main finding of our study is that CAm neurons also resemble COM neurons in their responsivity to $5-\mathrm{HT}$, whereby the vast majority of CAm neurons exhibit 2A-mediated responses, either in the form of purely excitatory responses to 5-HT (in 5-HT-excited neurons), or after transient 1A-mediated inhibition (in 5-HT-biphasic neurons). As with most other physiological measurements, serotonergic responses in CAm neurons were not laterality or sex-dependent (Table 3). Further, and as previously revealed in COM neurons (Stephens et al., 2014), 5-HT was unable to excite CAm neurons in the absence of additional excitatory drive, but was sufficient to promote AP generation in response to suprathreshold simulated synaptic 

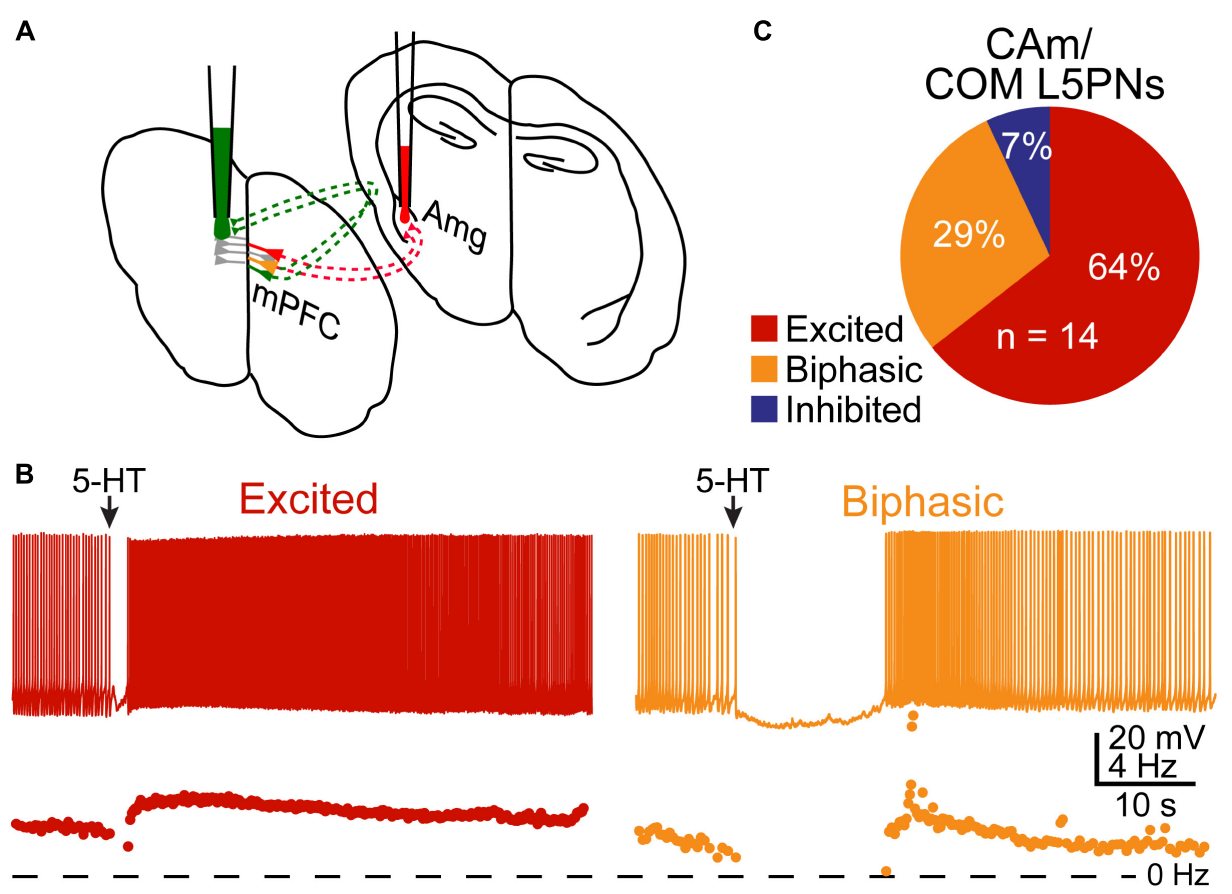

D
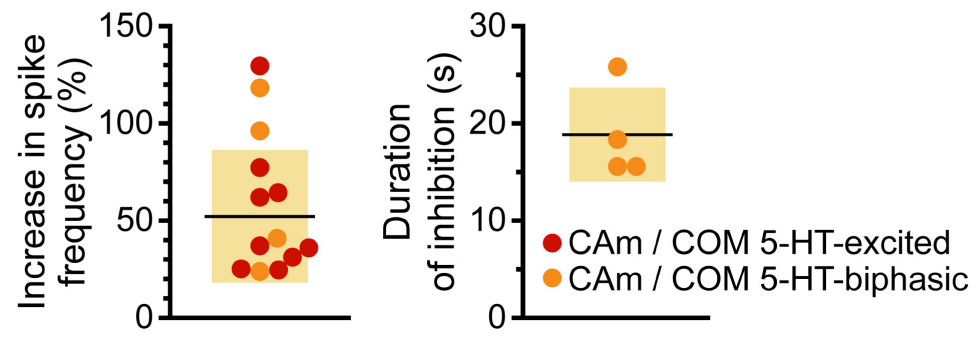

FIGURE 7 | Some CAm neurons are also COM neurons. (A) Diagram of dual-labeling of CAm/COM neurons in the medial prefrontal cortex. (B) Voltage traces (top) and ISF plots (bottom) for two double-labeled CAm/COM neurons exhibiting excitatory (red) or biphasic (yellow) responses to 5-HT. (C) Proportions of 14 CAm/COM double-labeled neurons exhibiting 5-HT responses. (D) Plots of the magnitudes of 5-HT excitatory responses in 5-HT-excited (red) and 5-HT-biphasic (yellow)

CAm/COM neurons (left) and the durations of inhibition in 5-HT-biphasic CAm/COM neurons (right).

input in 5-HT-excited neurons. This suggests that 5-HT may act to promote cortical output to the amygdala by enhancing the activity of subsets of $2 \mathrm{~A}$-expressing CAm neurons that are simultaneously receiving strong excitatory input.

In COM neurons, serotonergic excitation is mediated by $\mathrm{G}_{\mathrm{q}}$-coupled $2 \mathrm{~A}$ receptors that engage three postsynaptic ionic effectors to enhance cortical neuron excitability: inhibition of $\mathrm{K}_{\mathrm{V}} 7$ potassium channels and activation of two non-specific cation conductances (Stephens et al., 2018). Interestingly, these same ionic mechanisms underlie cholinergic excitation of CPn neurons in the prelimbic cortex (Baker et al., 2018), suggesting that they have a conserved role in $\mathrm{G}_{\mathrm{q}}$-mediated excitation across cortical neuron subtypes and neurotransmitter systems. On the other hand, serotonergic inhibition during biphasic responses results from $\mathrm{G}_{\mathrm{i} / \mathrm{o}}$-coupled $1 \mathrm{~A}$ receptors enhancing potassium conductances, including those mediated by G-proteingated inwardly rectifying potassium channels (Andrade and Nicoll, 1987; Luscher et al., 1997). Using specific antagonists, we confirmed that serotonergic excitation and inhibition of CAm neurons are mediated by the same $2 \mathrm{~A}$ and $1 \mathrm{~A}$ receptors (respectively) that gate serotonergic responses in COM neurons. While specific ionic mechanisms were not tested in this study, given the overlap in COM and CAm neuron populations, and the qualitative and quantitative similarities of their 5-HT responses, it would seem likely that 5-HT engages a similar set of mechanisms in both CAm and COM neurons.

\section{A Functional Role for Serotonergic Regulation of CAm Neurons}

CAm neurons in the prelimbic cortex contribute to reciprocal CAm circuits that are engaged during the expression of conditioned fear (Stevenson, 2011; Karalis et al., 2016), and fear conditioning is associated with release of 5-HT into the prelimbic cortex (Hashimoto et al., 1999; Bland et al., 2003). Our results demonstrate that CAm neurons are responsive 
to 5-HT, suggesting that 5-HT may influence the learning or expression of conditioned fear. Indeed, studies utilizing acute administration of SSRIs to globally increase extracellular 5-HT report enhanced acquisition and expression of cued (but not contextual) fear responses (for reviews, see Bauer, 2015; Bocchio et al., 2016). Further, pharmacological studies have revealed oppositional impact of $1 \mathrm{~A}$ and $2 \mathrm{~A}$ receptors in fear expression. For instance, systemic injection of a $2 \mathrm{~A}$ receptor agonist enhances freezing in response to conditioned cues in rodents (Zhang et al., 2013), while 1A agonists delivered systemically (Inoue et al., 1996; Youn et al., 2009; Ohyama et al., 2016), or locally into the prelimbic cortex (Almada et al., 2015), reduce contextual freezing responses and fear-potentiated startle, respectively. Consistent with a role for $2 \mathrm{~A}$ receptors in potentiating fear responses, systemic (Zhang et al., 2013) or local blockade of $2 \mathrm{~A}$ receptors in the prelimbic cortex (León et al., 2017) can impair the expression of conditioned fear.

Since prelimbic CAm neurons exhibit 2A-dependent excitation, 5-HT could contribute to the enhanced functional connectivity observed between the prelimbic cortex and the amygdala during fear behaviors (Karalis et al., 2016). However, the net effect of 5-HT on prelimbic output is difficult to predict because, while $2 \mathrm{~A}$ receptors may increase the excitability of CAm neurons, 5-HT also influences the excitability of cortical interneurons, and can act presynaptically to regulate glutamate and GABA release (for review, see Puig and Gulledge, 2011). Predictions of 5-HT effects on behavior are further complicated by potentially competing influences of CAm projections arising from different cortical areas (e.g., infralimbic vs prelimbic cortex; see, for instance, Vidal-Gonzalez et al., 2006), and from direct effects of 5-HT on the amygdala or other subcortical structures contributing to fear responses (Bocchio et al., 2016). However, future studies may surmount these limitations by employing genetically targeted approaches that allow for selective manipulation of specific neuron or 5-HT receptor subtypes in restricted brain regions (e.g., prelimbic cortex) at select

\section{REFERENCES}

Almada, R. C., Coimbra, N. C., and Brandao, M. L. (2015). Medial prefrontal cortex serotonergic and GABAergic mechanisms modulate the expression of contextual fear: intratelencephalic pathways and differential involvement of cortical subregions. Neuroscience 284, 988-997. doi: 10.1016/j.neuroscience. 2014.11.001

Andrade, R., and Nicoll, R. A. (1987). Pharmacologically distinct actions of serotonin on single pyramidal neurones of the rat hippocampus recorded in vitro. J. Physiol. 394, 99-124. doi: 10.1113/jphysiol.1987.sp016862

Avesar, D., and Gulledge, A. T. (2012). Selective serotonergic excitation of callosal projection neurons. Front. Neural Circuits 6:12. doi: 10.3389/fncir.2012.00012

Avesar, D., Stephens, E., and Gulledge, A. T. (2018). Serotonergic regulation of corticoamygdalar neurons in the mouse prelimbic cortex. bioRxiv [Preprint]. doi: $10.1101 / 293688$

Baker, A. L., O'toole, R. J., and Gulledge, A. T. (2018). Preferential cholinergic excitation of corticopontine neurons. J. Physiol. 596, 1659-1679. doi: 10.1113/ JP275194

Bauer, E. P. (2015). Serotonin in fear conditioning processes. Behav. Brain Res. 277, 68-77. doi: 10.1016/j.bbr.2014.07.028

Bland, S. T., Hargrave, D., Pepin, J. L., Amat, J., Watkins, L. R., and Maier, S. F. (2003). Stressor controllability modulates stress-induced dopamine and serotonin efflux and morphine-induced serotonin efflux in the medial developmental time points. Such studies, carried out at a systems level, may reveal a role for 5-HT in facilitating not only fear behaviors, but also in regulating an extended intratelencephalic circuit encompassing COM and CAm neurons in both cerebral hemispheres, amygdalar neurons, CA1 neurons in the ventral hippocampus, and their collective overlapping efferents to the striatum (Cho et al., 2013; Heilbronner et al., 2016), to more broadly regulate emotional learning, decision making, and behavior (Vertes, 2006).

\section{ETHICS STATEMENT}

All experiments were conducted according to methods approved by the Institutional Animal Care and Use Committee of Dartmouth College.

\section{AUTHOR CONTRIBUTIONS}

DA and AG designed the research. DA and ES performed the research and analyzed the data. AG wrote the manuscript.

\section{FUNDING}

This work was supported by a grant from the National Institute for Mental Health (R01 MH099054; AG) and a Frank and Myra Weiser Scholar Award (AG).

\section{ACKNOWLEDGMENTS}

The authors thank Saiko Ikeda for technical assistance and Arielle Baker for comments on the manuscript. A preprint of this manuscript is available on BioRxiv (Avesar et al., 2018).

prefrontal cortex. Neuropsychopharmacology 28, 1589-1596. doi: 10.1038/sj. npp. 1300206

Bocchio, M., Mchugh, S. B., Bannerman, D. M., Sharp, T., and Capogna, M. (2016). Serotonin, amygdala and fear: assembling the puzzle. Front. Neural Circuits 10:24. doi: 10.3389/fncir.2016.00024

Bravo-Rivera, C., Roman-Ortiz, C., Brignoni-Perez, E., Sotres-Bayon, F., and Quirk, G. J. (2014). Neural structures mediating expression and extinction of platform-mediated avoidance. J. Neurosci. 34, 9736-9742. doi: 10.1523/ JNEUROSCI.0191-14.2014

Bravo-Rivera, C., Roman-Ortiz, C., Montesinos-Cartagena, M., and Quirk, G. J. (2015). Persistent active avoidance correlates with activity in prelimbic cortex and ventral striatum. Front. Behav. Neurosci. 9:184. doi: 10.3389/fnbeh.2015. 00184

Burghardt, N. S., and Bauer, E. P. (2013). Acute and chronic effects of selective serotonin reuptake inhibitor treatment on fear conditioning: implications for underlying fear circuits. Neuroscience 247, 253-272. doi: 10.1016/j. neuroscience.2013.05.050

Burgos-Robles, A., Vidal-Gonzalez, I., and Quirk, G. J. (2009). Sustained conditioned responses in prelimbic prefrontal neurons are correlated with fear expression and extinction failure. J. Neurosci. 29, 8474-8482. doi: 10.1523/ JNEUROSCI.0378-09.2009

Cheriyan, J., Kaushik, M. K., Ferreira, A. N., and Sheets, P. L. (2016). Specific targeting of the basolateral amygdala to projectionally defined pyramidal 
neurons in prelimbic and infralimbic cortex. eNeuro 3:ENEURO.0002-16.2016. doi: 10.1523/ENEURO.0002-16.2016

Cho, Y. T., Ernst, M., and Fudge, J. L. (2013). Cortico-amygdala-striatal circuits are organized as hierarchical subsystems through the primate amygdala. J. Neurosci. 33, 14017-14030. doi: 10.1523/JNEUROSCI.0170-13.2013

Corcoran, K. A., and Quirk, G. J. (2007). Activity in prelimbic cortex is necessary for the expression of learned, but not innate, fears. J. Neurosci. 27, 840-844. doi: 10.1523/JNEUROSCI.5327-06.2007

Courtin, J., Bienvenu, T. C., Einarsson, E. O., and Herry, C. (2013). Medial prefrontal cortex neuronal circuits in fear behavior. Neuroscience 240, 219-242. doi: 10.1016/j.neuroscience.2013.03.001

Dejean, C., Courtin, J., Karalis, N., Chaudun, F., Wurtz, H., Bienvenu, T. C., et al. (2016). Prefrontal neuronal assemblies temporally control fear behaviour. Nature 535, 420-424. doi: 10.1038/nature18630

Dembrow, N. C., Chitwood, R. A., and Johnston, D. (2010). Projectionspecific neuromodulation of medial prefrontal cortex neurons. J. Neurosci. 30, 16922-16937. doi: 10.1523/JNEUROSCI.3644-10.2010

Do Monte, F. H., Quirk, G. J., Li, B., and Penzo, M. A. (2016). Retrieving fear memories, as time goes by. Mol. Psychiatry 21, 1027-1036. doi: 10.1038/mp. 2016.78

Euston, D. R., Gruber, A. J., and Mcnaughton, B. L. (2012). The role of medial prefrontal cortex in memory and decision making. Neuron 76, 1057-1070. doi: $10.1016 /$ j.neuron.2012.12.002

Ferreira, R., and Nobre, M. J. (2014). Conditioned fear in low- and high-anxious rats is differentially regulated by cortical subcortical and midbrain 5-HT(1A) receptors. Neuroscience 268, 159-168. doi: 10.1016/j.neuroscience.2014.03.005

Gabbott, P. L., Warner, T. A., Jays, P. R., Salway, P., and Busby, S. J. (2005). Prefrontal cortex in the rat: projections to subcortical autonomic, motor, and limbic centers. J. Comp. Neurol. 492, 145-177. doi: 10.1002/cne.20738

Hashimoto, S., Inoue, T., and Koyama, T. (1999). Effects of conditioned fear stress on serotonin neurotransmission and freezing behavior in rats. Eur. J. Pharmacol. 378, 23-30. doi: 10.1016/S0014-2999(99)00441-0

Heilbronner, S. R., Rodriguez-Romaguera, J., Quirk, G. J., Groenewegen, H. J., and Haber, S. N. (2016). Circuit-based corticostriatal homologies between rat and primate. Biol. Psychiatry 80, 509-521. doi: 10.1016/j.biopsych.2016.05.012

Inoue, T., Tsuchiya, K., and Koyama, T. (1996). Serotonergic activation reduces defensive freezing in the conditioned fear paradigm. Pharmacol. Biochem. Behav. 53, 825-831. doi: 10.1016/0091-3057(95)02084-5

Karalis, N., Dejean, C., Chaudun, F., Khoder, S., Rozeske, R. R., Wurtz, H., et al. (2016). 4-Hz oscillations synchronize prefrontal-amygdala circuits during fear behavior. Nat. Neurosci. 19, 605-612. doi: 10.1038/nn.4251

León, L. A., Castro-Gomes, V., Zárate-Guerrero, S., Corredor, K., Mello Cruz, A. P., Brandão, M. L., et al. (2017). Behavioral effects of systemic, infralimbic and prelimbic injections of a serotonin 5-HT2A antagonist in carioca high- and lowconditioned freezing rats. Front. Behav. Neurosci. 11:117. doi: 10.3389/fnbeh. 2017.00117

Little, J. P., and Carter, A. G. (2013). Synaptic mechanisms underlying strong reciprocal connectivity between the medial prefrontal cortex and basolateral amygdala. J. Neurosci. 33, 15333-15342. doi: 10.1523/JNEUROSCI.2385-13. 2013

Luscher, C., Jan, L. Y., Stoffel, M., Malenka, R. C., and Nicoll, R. A. (1997). G protein-coupled inwardly rectifying $\mathrm{K}+$ channels (GIRKs) mediate postsynaptic but not presynaptic transmitter actions in hippocampal neurons. Neuron 19, 687-695. doi: 10.1016/S0896-6273(00)80381-5

Mcdonald, A. J., Mascagni, F., and Guo, L. (1996). Projections of the medial and lateral prefrontal cortices to the amygdala: a Phaseolus vulgaris leucoagglutinin study in the rat. Neuroscience 71, 55-75. doi: 10.1016/0306-4522(95)00417-3

Ohyama, M., Kondo, M., Yamauchi, M., Imanishi, T., and Koyama, T. (2016). Asenapine reduces anxiety-related behaviours in rat conditioned fear stress model. Acta Neuropsychiatr. 28, 327-336. doi: 10.1017/neu.2016.17

Orsini, C. A., Moorman, D. E., Young, J. W., Setlow, B., and Floresco, S. B. (2015). Neural mechanisms regulating different forms of risk-related decisionmaking: insights from animal models. Neurosci. Biobehav. Rev. 58, 147-167. doi: 10.1016/j.neubiorev.2015.04.009

Paxinos, G., and Franklin, K. B. J. (2004). The Mouse Brain in Stereotaxic Coordinates. San Diego, CA: Elsevier Academic Press.
Puig, M. V., and Gulledge, A. T. (2011). Serotonin and prefrontal cortex function: neurons, networks, and circuits. Mol. Neurobiol. 44, 449-464. doi: 10.1007/ s12035-011-8214-0

Ragozzino, M. E. (2007). The contribution of the medial prefrontal cortex, orbitofrontal cortex, and dorsomedial striatum to behavioral flexibility. Ann. N. Y. Acad. Sci. 1121, 355-375. doi: 10.1196/annals.1401.013

Ravinder, S., Burghardt, N. S., Brodsky, R., Bauer, E. P., and Chattarji, S. (2013). A role for the extended amygdala in the fear-enhancing effects of acute selective serotonin reuptake inhibitor treatment. Transl. Psychiatry 3:e209. doi: 10.1038/ tp.2012.137

Sierra-Mercado, D., Padilla-Coreano, N., and Quirk, G. J. (2011). Dissociable roles of prelimbic and infralimbic cortices, ventral hippocampus, and basolateral amygdala in the expression and extinction of conditioned fear. Neuropsychopharmacology 36, 529-538. doi: 10.1038/npp.2010.184

Song, C., Ehlers, V. L., and Moyer, J. R. Jr. (2015). Trace fear conditioning differentially modulates intrinsic excitability of medial prefrontal cortexbasolateral complex of amygdala projection neurons in infralimbic and prelimbic cortices. J. Neurosci. 35, 13511-13524. doi: 10.1523/JNEUROSCI. 2329-15.2015

Stephens, E. K., Avesar, D., and Gulledge, A. T. (2014). Activity-dependent serotonergic excitation of callosal projection neurons in the mouse prefrontal cortex. Front. Neural Circuits 8:97. doi: 10.3389/fncir.2014.00097

Stephens, E. K., Baker, A. L., and Gulledge, A. T. (2018). Mechanisms underlying serotonergic excitation of callosal projection neurons in the mouse medial prefrontal cortex. Front. Neural Circuits 12:2. doi: 10.3389/fncir.2018. 00002

Stevenson, C. W. (2011). Role of amygdala-prefrontal cortex circuitry in regulating the expression of contextual fear memory. Neurobiol. Learn. Mem. 96, 315-323. doi: 10.1016/j.nlm.2011.06.005

Varela, J. A., Wang, J., Christianson, J. P., Maier, S. F., and Cooper, D. C. (2012). Control over stress, but not stress per se increases prefrontal cortical pyramidal neuron excitability. J. Neurosci. 32, 12848-12853. doi: 10.1523/JNEUROSCI. 2669-12.2012

Vertes, R. P. (2004). Differential projections of the infralimbic and prelimbic cortex in the rat. Synapse 51, 32-58. doi: 10.1002/syn.10279

Vertes, R. P. (2006). Interactions among the medial prefrontal cortex, hippocampus and midline thalamus in emotional and cognitive processing in the rat. Neuroscience 142, 1-20. doi: 10.1016/j.neuroscience.2006.06.027

Vidal-Gonzalez, I., Vidal-Gonzalez, B., Rauch, S. L., and Quirk, G. J. (2006). Microstimulation reveals opposing influences of prelimbic and infralimbic cortex on the expression of conditioned fear. Learn. Mem. 13, 728-733. doi: 10.1101/lm.306106

Yoshioka, M., Matsumoto, M., Togashi, H., and Saito, H. (1995). Effects of conditioned fear stress on 5-HT release in the rat prefrontal cortex. Pharmacol. Biochem. Behav. 51, 515-519. doi: 10.1016/0091-3057(95)00045-X

Youn, J., Misane, I., Eriksson, T. M., Millan, M. J., Ogren, S. O., Verhage, M., et al. (2009). Bidirectional modulation of classical fear conditioning in mice by $5-\mathrm{HT}(1 \mathrm{~A})$ receptor ligands with contrasting intrinsic activities. Neuropharmacology 57, 567-576. doi: 10.1016/j.neuropharm.2009. 07.011

Zhang, G., Asgeirsdottir, H. N., Cohen, S. J., Munchow, A. H., Barrera, M. P., and Stackman, R. W. Jr. (2013). Stimulation of serotonin 2A receptors facilitates consolidation and extinction of fear memory in C57BL/6J mice. Neuropharmacology 64, 403-413. doi: 10.1016/j.neuropharm.2012.06.007

Conflict of Interest Statement: The authors declare that the research was conducted in the absence of any commercial or financial relationships that could be construed as a potential conflict of interest.

Copyright (C) 2018 Avesar, Stephens and Gulledge. This is an open-access article distributed under the terms of the Creative Commons Attribution License (CC BY). The use, distribution or reproduction in other forums is permitted, provided the original author(s) and the copyright owner(s) are credited and that the original publication in this journal is cited, in accordance with accepted academic practice. No use, distribution or reproduction is permitted which does not comply with these terms. 